\title{
Developmental expression and cellular distribution of Müllerian inhibiting substance in the primate ovary
}

\author{
Deepak Modi ${ }^{1,2}$, Deepa Bhartiya ${ }^{1,2}$ and Chander Puri ${ }^{2}$ \\ ${ }^{1}$ Cell Biology Department, Research Laboratory, BJ Wadia Hospital for Children, AD Marg, Parel, Mumbai 400012, \\ India and ${ }^{2}$ National Institute for Research in Reproductive Health, Indian Council of Medical Research, J M Street, \\ Parel, Mumbai 400 012, India
}

Correspondence should be addressed to D Modi; Email: deepaknmodi@yahoo.com, modidn@icmr.org.in

\begin{abstract}
Ovarian follicle formation during development and follicle maturation in adulthood are crucial determinants of female fertility and disruptions in these processes may result in subfertility or infertility. Among the several factors that are involved in ovarian physiology, Müllerian inhibiting substance (MIS) also known as anti-Müllerian hormone has emerged as an important marker to predict the follicle reserve. However, the roles of MIS in human ovarian physiology are unknown. To gain an insight into the potential roles of MIS in human ovarian differentiation during development and its regulation in adulthood, the expression profiles of MIS mRNA in the developing and adult human and monkey ovaries was examined by in situ hybridization. The results revealed that in the fetal human ovaries, MIS is specifically expressed at low levels in the granulosa cells of the developing primordial follicles; a small subset ( $\sim 2-3 \%$ ) of oocytes express high amounts of MIS. In the adult human and monkey ovary, MIS mRNA is expressed at low levels in the primordial follicles, maximally in the primary and secondary follicles, and the expression is downregulated in the antral and atetric follicles. MIS expression is extinguished in the granulosa cells only after ovulation. These observations strongly favor the regulatory roles of MIS in folliculogenesis. MIS in the primate ovary may exert its effect during the primordial follicle formation to the terminal granulosa cell differentiation. The presence of MIS in a small subset of oocytes in the fetal ovary further points towards its additional role during fetal oocyte development.
\end{abstract}

Reproduction (2006) 132 443-453

\section{Introduction}

The process of ovarian follicle growth and differentiation is a dynamically controlled event involving initial recruitment of the follicles from the primordial pool followed by a phase of growth. The growing follicles are next selected for differentiation to ovulate or undergo atresia (McGee \& Hsueh 2000). This cyclic process of follicle selection unto ovulation or atresia is regulated and is under strict endocrine, autocrine, and paracrine control.

Among the several local factors involved in follicular differentiation, Müllerian inhibiting substance (MIS) also known as anti-Müllerian hormone $(\mathrm{AMH})$ has been demonstrated to play a crucial role in the regulation of folliculogenesis in rodents. Essentially a male hormone, produced by the Sertoli cells of the fetal testis (Lee \& Donahoe 1993, Lee et al. 1994), MIS mRNA and protein has been detected in the granulosa cells of the adult rodent ovary. The expression is specifically detected in the early preantral and antral follicles; the expression is lost in the ovulatory or the atetric follicles (Uneo et al. 1989a, Hirobe et al. 1992, Durlinger et al. 2002a,b). Interestingly, targeted disruption of MIS in female mice leads to rapid depletion of the primordial follicles (Durlinger et al. 1999). The ovaries of these mice have more preantral and small antral follicles as compared with wild-type littermates. In addition, the follicles of MIS knockout mice demonstrate altered sensitivity to FSH (Durlinger et al. 2001). Based on these studies, it has been suggested that MIS may play a role in follicle selection in the rodent ovary (Durlinger et al. 2002a, Visser et al. 2006).

In human females, circulatory levels of MIS have been found to correlate with the ovarian reserve, the levels are altered in cases of abnormal folliculogenesis (Fallat et al. 1997, Cook et al. 2002, Penarrubia et al. 2005, Piltonen et al. 2005, van Rooij et al. 2005, reviewed in Visser et al. 2006) suggesting a role of MIS in human ovarian functions. However, the roles of MIS in human ovary have not been well defined. Limited and controversial information exist on the expression profiles of MIS in the human ovary. During development, MIS protein has 
been demonstrated to express in the human fetal ovary only in the primary follicles late in gestation; however, MIS mRNA has been reportedly detected even at 13 weeks of development (Voutilainen \& Miller 1987, Rajpert-De Meyets et al. 1999). In the adult ovary, expression of immunoreactive MIS has been restricted to the granulosa cells of primary, secondary, preantral, and antral follicles; the expression is downregulated in the atetric follicles. The granulosa cells of the primordial follicles, the oocytes, or the ovarian stroma do not stain positive for MIS (Weenen et al. 2004). Contrasting these observations, in a recent study, immunoreactive MIS has been detected in the oocytes, the ovarian stroma and the thecal cells along with the granulosa cells of the growing follicles (Stubbs et al. 2005). These observations differ from those reported in rodents where MIS mRNA or protein has not been detected in the fetal ovary, primordial follicles, oocytes, or the interstitial cells of the adult rat ovary (Uneo et al. 1989a, Hirobe et al. 1992, Baarends et al. 1995, Durlinger et al. 2002a). In murine system, the expression of MIS is identical in all the follicles of the same class (Uneo et al. 1989a, Hirobe et al. 1992). However, in the human ovary, heterogeneity has been reported in the expression MIS protein in the granulosa cells of the developing follicles, particularly the antral follicles (Weenen et al. 2004, Stubbs et al. 2005). The differences in the pattern of MIS expression between species and the contrasting observations reported in human studies makes it difficult to speculate the precise roles of MIS in ovarian physiology.

To gain better insight into the roles of MIS in the process of follicle formation, growth, and differentiation, particularly in the primates, the detailed ontogeny of MIS mRNA expression in the developing and the adult human ovary was examined. Additionally, the pattern of MIS mRNA expression in the ovary of adult cyclic bonnet monkeys is also compared, to determine speciesspecific changes, if any, that may occur. We chose to study the mRNA expression of MIS rather than protein, as it would permit us to exactly determine the site of protein synthesis and decide, if MIS expression during folliculogenesis is regulated transcriptionally. The aim of the study is to examine the detailed cellular localization and expression pattern of MIS mRNA in the process of follicular development prenatally and during adult folliculogenesis in primates.

\section{Material and Methods}

The present study was approved by the local ethics committee. All tissues were collected after informed consent. All the samples were anonymous retaining only the essential clinical information.

Human fetal ovaries were collected from secondtrimester, legal abortions done at the Obstetric and Gynecology Department of King Edward Memorial Hospital (KEMH). The details of collection of these gonads and anthropometric analysis have been reported earlier (Modi et al. 2003).

Fetal samples: Twenty-eight second-trimester fetal ovaries (13-23 weeks of gestation) were analyzed in the present study. Somatic tissues from two male and two female fetuses aged 20 weeks were also collected.

Neonatal samples: Ovaries were obtained from two female neonates that had expired soon after birth and were undergoing autopsy to determine the cause of death.

Adult samples: Adult human ovaries were collected from three women (33-39 years) undergoing panhysterectomy for reasons other than ovarian disorders.

To examine the expression profile of MIS mRNA in the granulosa cells post-ovulation, two cumulous oocyte complexes (COCs) were obtained from a woman undergoing superovulation and oocyte retrieval for in vitro fertilization owing to male factor infertility. These were kindly donated for research purposes and collected from the Malpani Infertility Clinic (Mumbai, India). The protocol of superovulation and oocyte retrieval has been detailed previously (Malpani et al. 2002).

Monkey ovaries: Ovaries from three adult regularly cycling bonnet monkeys (Macaca radiata) were collected in the late follicular phase. The cyclicity and the hormonal profiles of the animals have been reported previously (Patil et al. 2005).

\section{Tissue processing}

The tissue was immediately fixed in $10 \%$ buffered formaldehyde for 48-96 $\mathrm{h}$ and processed for routine paraffin embedding and sectioning. Sections of $5 \mu \mathrm{m}$ thickness were mounted on aminosailine-coated glass slides air-dried and stored in dust-free boxes at room temperature until use. Care was taken at all stages to avoid RNase contamination. The COCs were directly fixed on glass slides, air dried, and immediately fixed in $4 \%$ buffered formaldehyde for $20 \mathrm{~min}$, dehydrated in alcohol, and stored until use.

\section{In situ hybridization}

Expression of MIS transcripts was studied in the ovaries using non-radioactive in situ hybridization (ISH). All the reagents were purchased from Roche and Sigma. All glass and plastic wares used were autoclaved and baked; all solutions and water were treated with diethylpyrocarbonate (DEPC) to inactivate RNase prior to use. The general protocol for ISH has been reported previously (Modi et al. 2005, Shah et al. 2005).

\section{Probe preparation}

The probe used for in situ hybridization was an oligonucleotide (5'-CGGTAGGCGCCTTGAGCTCCTCAGCGG-3') commercially synthesized (Gibco) and labeled with Digoxygenein (Dig) using the $3^{\prime}$ tailing kit 
according to the manufacturer's instructions (Roche). The specificity of the probe sequence was established by examining its homology with other sequences in the database. All database searches were carried out using the BLAST search engine at www.ncbi.nlm.nih.gov at low stringency.

\section{Hybridization}

For ISH, the sections were deparaffinized in xylene, hydrated, and refixed in $2 \%$ paraformaldehyde for $10 \mathrm{~min}$. The COCs were directly refixed in paraformaldehyde for $5 \mathrm{~min}$ and processed further. After washing in $0.1 \mathrm{M} \mathrm{PBS}(\mathrm{pH} 7.0)$, the slides were incubated in $2 \times$ SSC $(1 \times \mathrm{SSC}=0.15 \mathrm{M}$ sodium chloride and $0.015 \mathrm{M}$ sodium citrate, $\mathrm{pH}$ 7) for $15 \mathrm{~min}$ at room temperature. Prehybridization was carried out at room temperature for $1 \mathrm{~h}$ in a pre-hybridization cocktail containing $50 \%$ formamide, $4 \times$ SSC, $5 \times$ Denharts solution, $0.25 \%$ yeast tRNA, $0.5 \%$ sheared Salmon sperm DNA, and $10 \%$ dextran sulphate. After pre-hybridization, the sections were hybridized overnight at $56{ }^{\circ} \mathrm{C}$ with the labeled probe diluted in the same cocktail at a concentration of $2-5 \mathrm{pmol} / \mu \mathrm{l}$. The sections were stringently washed in varying concentrations of SSC containing $0.1 \%$ Tween 20 ( $4 \times$ SSC, 20 min twice; $2 \times$ SSC, 20 min twice; $1 \times$ SSC, $20 \mathrm{~min}$ twice; $0.5 \times$ SSC $15 \mathrm{~min}$ twice; and $0.1 \times$ SSC $15 \mathrm{~min}$ once) followed by blocking for $2 \mathrm{~h}$ at room temperature in blocking solution containing $2 \%$ normal sheep serum, $0.1 \%$ Triton X-100 in $0.1 \mathrm{M}$ Tris- $\mathrm{HCl}$ buffer $(\mathrm{pH}$ 7.5). The washing stringency described above was derived after extensive experimentation using human fetal testicular tissue. This stringency-permitted detection of the signals only in the Sertoli cells, increasing the wash stringency above this (by either increasing the temperature or reducing the salt concentration) lead to loss of signals from the Sertoli cells.

After blocking, the sections were incubated overnight at $4{ }^{\circ} \mathrm{C}$ in alkaline phosphatase-conjugated anti-Dig antibody diluted (1:500) in the above blocking solution. The slides were then extensively washed in $0.1 \mathrm{M}$ Tris- $\mathrm{HCl}(\mathrm{pH} 7.5)$ and equilibrated in $0.1 \mathrm{M}$ Tris- $\mathrm{HCl}$ (pH 9.5) for $10 \mathrm{~min}$. Detection was carried out at $\mathrm{pH} 9.5$ for $35 \mathrm{~min}$ at room temperature in a solution of nitro blue tetrazolium and 5-bromo-4-chloro-2-indoyl phosphate containing $0.2 \%$ levamisole as described previously (Modi et al. 2003) and mounted in aquamount. The sections were viewed light microscopically and representative areas were photographed using Olympus BX-60 microscope (Japan). The sections incubated using a sense probe served as negative controls and fetal testicular sections were used as positive control.

\section{Semiquantative analysis}

The ovarian sections were scored semiquantatively for extent of MIS expression by two independent observers.
An arbitrary score was given: 0, no expression; 1, negligible, but detectable expression; 2, mild expression; 3, moderate expression; and 4, strong expression. The final score was calculated as $\Sigma$ grade $\times$ number of cells counted/total number of cells. Values are expressed as mean \pm s.E.M. and statistical analysis was performed using Student's $t$-test.

\section{Follicular nomenclature}

The follicles were grouped according to the standard classification. Briefly, follicles with a single layer of flat granulosa cells were termed primordial follicles and those with a single layer of cuboidal granulosa cells as primary follicles. The follicles with more than one layer of granulosa cells and a well-defined theca layer were termed secondary or preantral follicles. Follicles containing an antral cavity were antral follicles and those follicles with a large antral cavity were termed ovulatory follicles. Follicles containing the granulosa cells scattered in the antral cavity and having degenerative changes were termed atretic follicles.

\section{Results}

The probe used in this study was highly specific to MIS, since the sequence did not share significant homology $(>50 \%$ ) with any of the other sequences in the database. MIS mRNA was visualized as brown to purple staining in the nucleus and cytoplasm of all positive samples. MIS transcripts were abundantly localized to the nucleus and cytoplasm of the Sertoli cells, but not in germ cells or other somatic cells of the human fetal testis (Fig. 1G). The specificity of staining is further evident by the absence of any signals in fetal somatic tissues like kidneys, heart, membranes, liver, lungs, placenta, and umbilical cord (data not shown).

\section{MIS expression in the developing human ovary}

MIS transcripts were consistently detected in all the ovarian samples examined from 13 to 23 weeks of development (Fig. 1A-F). However, the expression was at lower levels than that observed in the fetal testis (Fig. 1G). In the early developing ovary at 13-15 weeks of gestation, MIS transcripts were seen only in the cytoplasm and the nucleus of pregranulosa/somatic cells, the germ cells were MIS negative (Fig. 1B). As the development progressed (16-18 weeks), MIS-positive granulosa cells enveloped the MIS-negative oocytes marking the initiation of folliculogenesis (Fig. 1C and D). At 20 weeks and above, a large number of oocytes surrounded by MIS-positive granulosa cells (developing primordial follicles) were observed all over the ovarian parenchyma; naked oocytes not surrounded by groups of MIS-positive somatic cells were also detectable (Fig. 1E and F). As the development progressed, most oocytes formed 

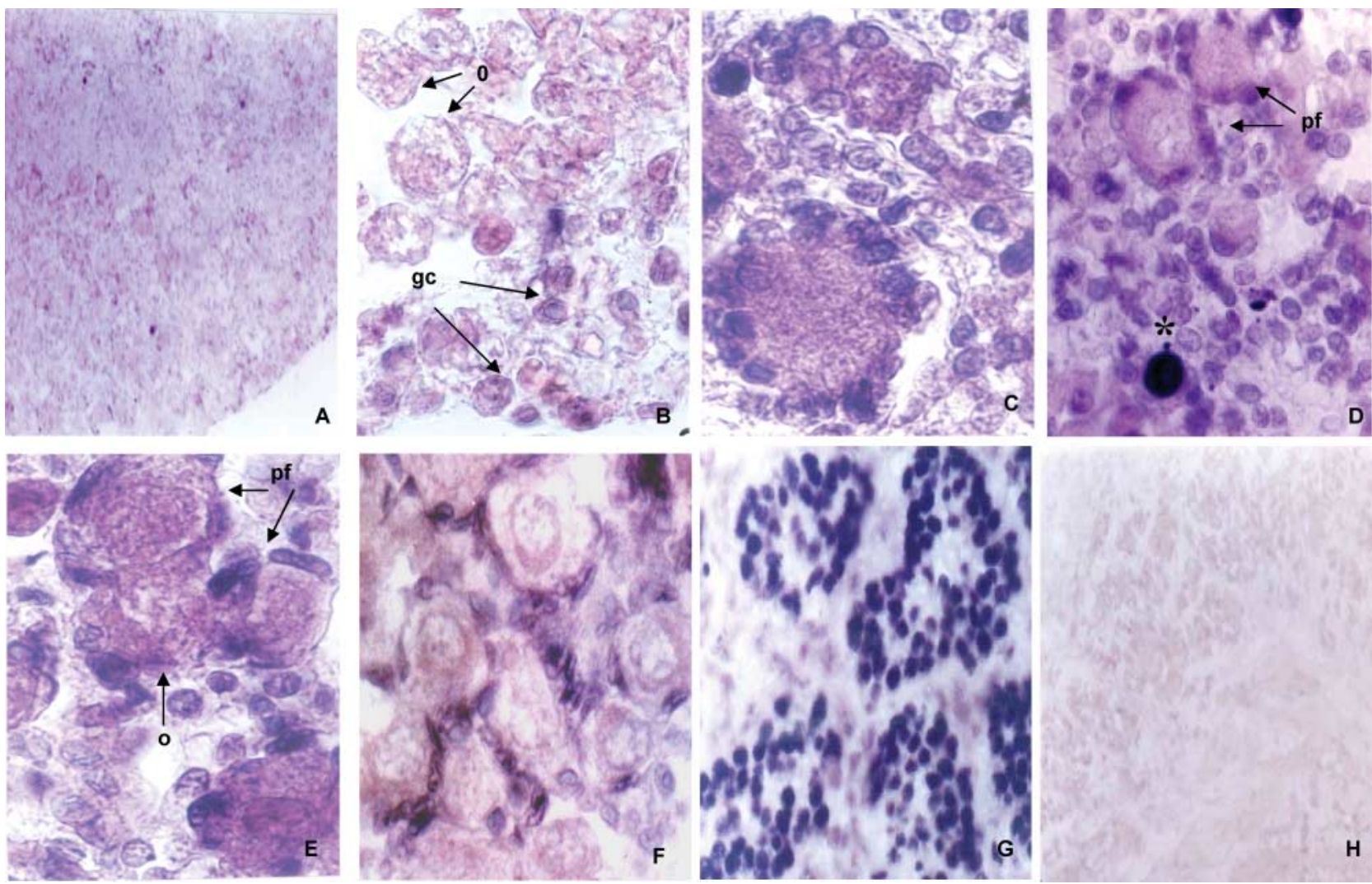

Figure 1 Expression of Müllerian inhibiting substance (MIS) mRNA in the developing human ovary. A is in situ hybridized 18-week-old fetal ovary, B is fetal ovary at 13 weeks showing week expression in the somatic (presumably pregranulosa) cells (gc); the oocytes (o) are MIS negative. C is ovary of a fetus at 16 weeks showing a developing follicle. D and $\mathrm{E}$ are ovaries at 18 and 20 weeks of development respectively showing developing primordial follicles (pf) and naked oocytes (o). Oocyte showing strong MIS expression is marked $\left(^{*}\right)$ in D. F is fetal ovary at 23 weeks of development showing a group of well-defined primordial follicles. $\mathrm{G}$ is fetal testis at 16 weeks of gestation as positive control. L is 16-week-old fetal ovary hybridized with a sense probe. Magnifications A $10 \times$, B-F $100 \times$ and digitized, G and H $20 \times$.

primordial follicles and MIS was detectable in the granulosa cells. At 23 weeks of development, along with the primordial follicles a single primary follicle was observed, MIS expression was higher in the granulosa cells of the primary follicle as compared with that in the primordial follicles (not shown).

Although most of the oocytes/oogonia were MIS negative, strong MIS mRNA expression was observed occasionally in some of the germ cells in the developing ovary (Fig. 1D). These cells had MIS expression nearly similar to that observed in the Sertoli cells of the fetal testis. The cells occurred at a frequency ranging from 1 to $3 \%$ with no appreciable difference between 13 and 23 weeks of development. Interestingly, the MIS-positive oocytes were generally not surrounded by the layer of somatic cells. The negative control did not show any staining (Fig. $1 \mathrm{H}$ ).

The semiquantative analysis of MIS mRNA expression in the granulosa cells was performed to evaluate if MIS mRNA expression in the ovary is regulated developmentally in the second trimester. As evident from Fig. 2A, no significant difference is noted in the expression of MIS in the fetal ovaries during the second trimester.

\section{MIS mRNA expression in the neonatal ovary}

In the newborn ovary, MIS expression was observed only in the granulosa cells of the primordial follicles at levels similar to those observed in the fetal ovary (Fig. 3A). None of the oocytes were MIS positive. Occasionally, MIS mRNA was detected in the stromal cells of the neonatal ovary.

In one of the neonatal ovary, some primary, secondary, and small antral follicles were detected along with the primordial follicles (Fig. 3B). MIS transcripts were observed in the granulosa cells of all these follicles, and the levels of MIS expression varied with the stages of folliculogenesis (Fig. 3B and C). As compared with the primordial follicles, MIS mRNA increased significantly in the granulosa cells of the primary and the secondary follicles. The small antral follicles had heterogeneous pattern of MIS mRNA expression with some of the granulosa cells being strongly positive for MIS mRNA whereas the others were MIS negative. In all, 90 follicles in different stages were analyzed semiquantitatively for the MIS expression levels in the granulosa cells (Fig. 2B). MIS expression increased significantly in the primary and the secondary follicles as compared with primordial 

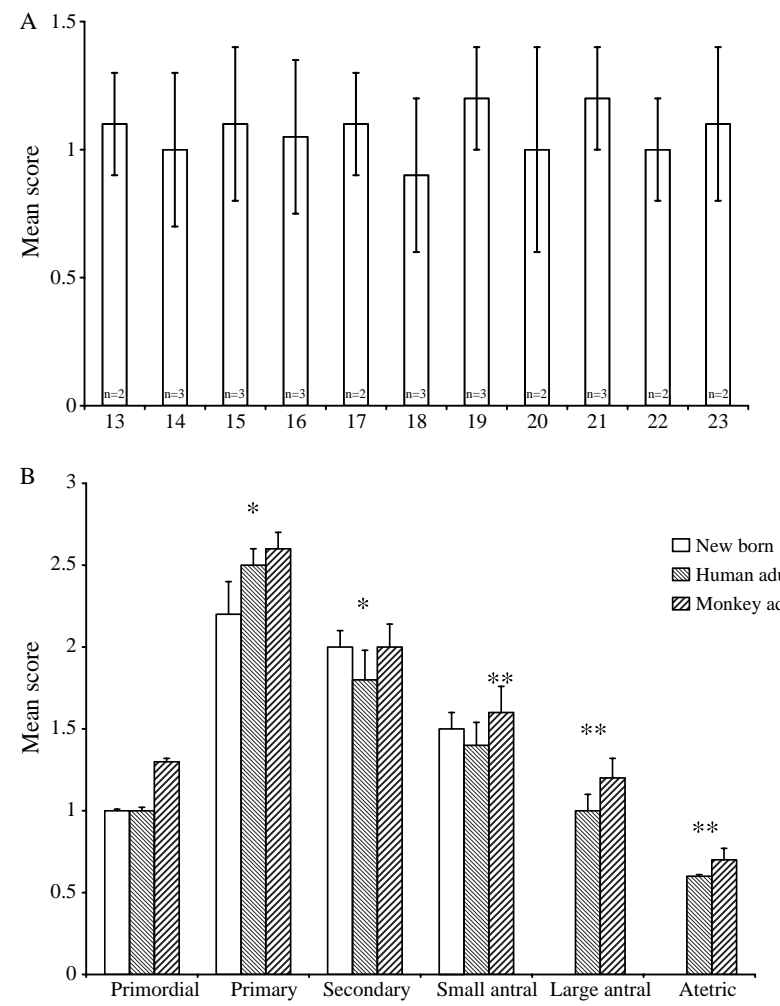

Figure 2 Semiquantitative assessment of Müllerian inhibiting substance (MIS) mRNA expression in human fetal neonatal and adult ovary. (A) Analysis of 28 fetal ovaries (n) between 13 and 23 weeks of gestation. (B) Analysis of MIS expression in the granulosa cells of the developing follicles from ovaries of a neonate, adult humans, and monkeys. Data is expressed as mean score \pm S.E.M. for the intensity of MIS expression. *Significantly different $(P<0.05)$ as compared to primordial follicles. ${ }^{*}$ Significantly different $(P<0.05)$ as compared to primary follicles.

follicles (Fig. 2B). MIS expression was lower in the small antral follicles as compared with the secondary or preantral follicles (Fig. 2B). No staining was visible in the negative control (Fig. 3D).

\section{MIS mRNA expression in the adult human ovary}

The results of MIS mRNA staining in the human ovary and the results of semiquantative analysis are represented in Figs 4 and 2B respectively. In all, 108 follicles from three adult ovaries were analyzed. MIS was specifically expressed in the granulosa cells of the follicles; the oocyte, theca cells, and the corpus luteum were generally negative for MIS mRNA. Occasionally, in some areas, the ovarian stroma showed presence of MIS transcripts.

The granulosa cells of the primordial follicles had low levels of MIS mRNA; the intensity of staining was highest in the primary and the preantral follicles (Fig. 4A-C). However, as the follicle size increased with the formation of the antrum, MIS mRNA expression reduced. The granulosa cells of the small antral follicles had reduced MIS mRNA expression as compared with the preantral follicles, the intensity of staining and the number of cells expressing MIS reduced further in the large antral follicles (Fig. 4D). By semiquantative analysis (Fig. 2B), MIS mRNA expression was found to be significantly lower in the small antral follicles as compared with secondary follicles, the expression of MIS reduced as the antral cavity enlarged. The mean staining intensity of MIS mRNA in large antral follicles was similar to the primordial follicles. MIS transcripts were minimal in the atetric follicles and in corpora lutea (Fig. 4G and $\mathrm{H}$ ).

In the large antral follicles, differences in the levels of MIS transcripts were observed in the cumulous cells as compared with the mural ones. In these follicles, the cumulous cells had MIS expression higher than the mural cells (Fig. 4E and F). However, the differences were not statistically significant.

To determine if MIS expression still persisted or extinguished in the cumulous cells, post-ovulation, in situ hybridization was performed on COCs obtained from a woman undergoing IVF for male factor infertility. MIS transcripts were detected in the granulosa cells of the COCs the oocyte was MIS negative (Fig. 4I-K). Interestingly, closer examination revealed that the transcripts were exclusively localized to the cytoplasm and the nuclear staining was absent in most of the cells (Fig. 4J and K). The negative control is shown in Fig. 4L.

\section{MIS expression in the monkey ovary}

The results of in situ hybridization for MIS in the monkey ovary are represented in Fig. 5 and the semiquantative analysis of gene expression is shown in Fig. 2B. The profiles of MIS expression in the ovaries of bonnet monkeys were identical to that observed in human ovary. MIS mRNA was detected in the granulosa cells of the growing follicles; the oocyte, theca cells, and the corpus luteum were negative for MIS mRNA. Occasionally, the ovarian stroma showed presence of MIS transcripts.

As seen in the human ovary, MIS expression increased in the primary and secondary follicles as compared with the primordial ones (Fig. 5A). As the antral cavity formed, the expression of MIS was downregulated in some granulosa cells (Fig. 5B). In large antral follicles, the number of granulosa cells with MIS expression reduced further (Fig. 5C). MIS expression was minimal in the ovulatory follicles (Fig. 5D). Differences were observed in the levels of MIS expression in the mural versus the cumulous granulosa cells. While the mural granulosa cells had low levels of MIS transcripts, the cumulous cells had relatively higher expression (Fig. 5G and H). MIS transcripts were negligible in the atretic follicles 

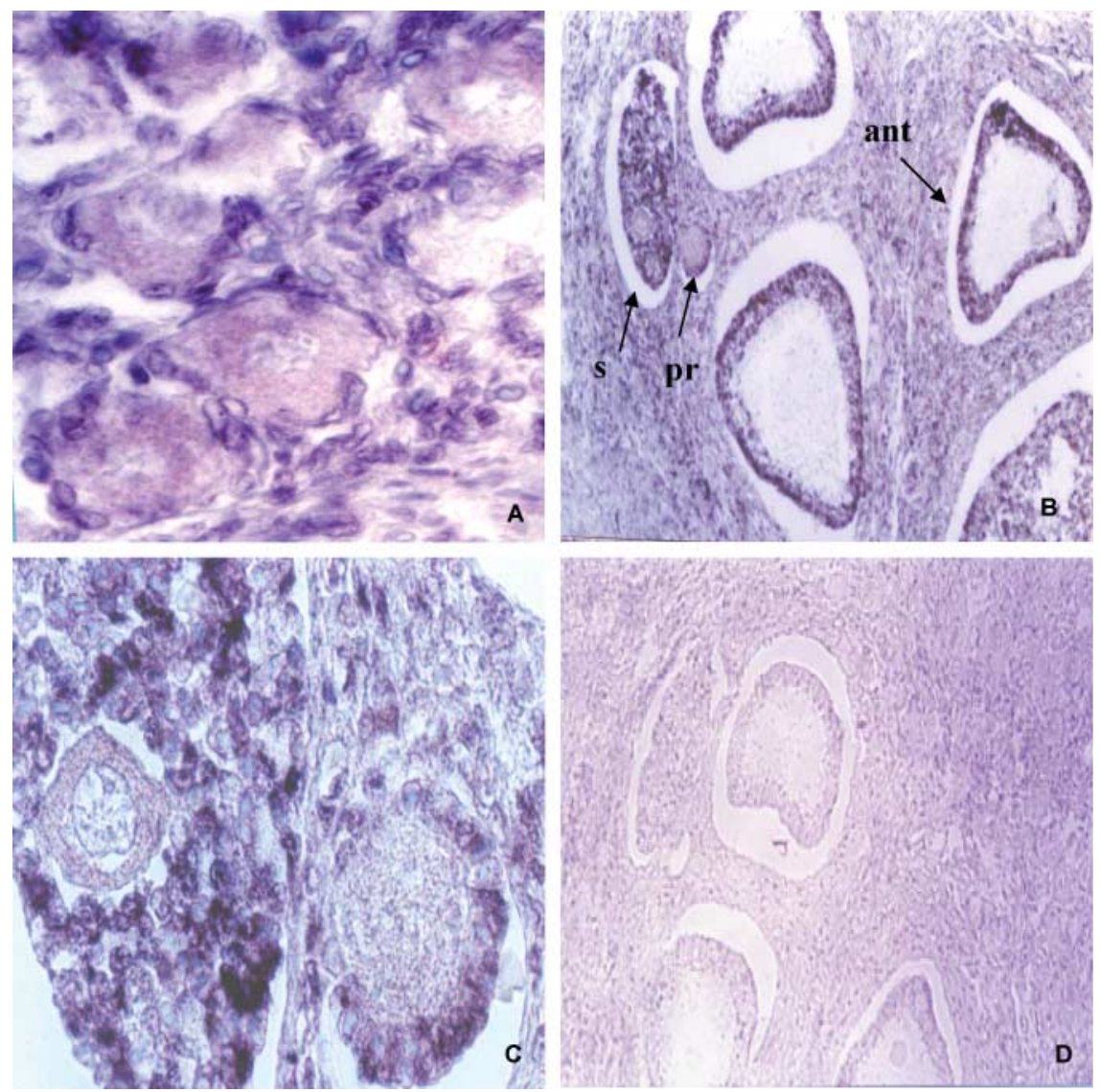

Figure 3 Müllerian inhibiting substance (MIS) mRNA in the neonatal ovary. (A) Newborn human ovary containing primordial follicles showing MIS expression in the granulosa cells of primordial follicles. (B) Expression of MIS in the growing follicles in a newborn ovary. Note the increase in expression in the primary (pr) and secondary (s) follicles, and a marginal drop in the antral (ant) follicles. (C) Enlarged view of the primary and large secondary follicles. (D) Negative control. Magnifications A $100 \times$, B and D $20 \times, C 40 \times$ and digitized.
(Fig. 5E and F). The negative control did not show any appreciable staining. (Fig. 5I).

In all, 228 follicles from ovaries of three different animals were analyzed. In the monkey ovary, the granulosa cells of the primordial follicles had very low levels of MIS mRNA; the expression increased and was maximal in the primary follicles (Fig. 4A). Nearly all the granulosa cells of the primary follicles stained positive for MIS mRNA, the expression increased by almost twofold over the primordial follicles.

As the follicular development proceeded, the expression of MIS was maximal in all the granulosa cells of the preantral follicles. The strongest staining for MIS was apparent in the preantral follicles that had 2-4 layers of granulosa cells (Fig. 4A). Semiquantative analysis reveled MIS expression to be marginally lower in the preantral follicles as compared with the primary follicles, although the differences were not significant.

Among the small antral follicles, in the follicles that had small antral cavity, the expression was similar to the secondary follicles (Fig. 4B), the intensity of staining and the number of cells expressing MIS reduced in the follicles with larger antrum (Fig. 4C). By semiquantative analysis, MIS mRNA expression was found to be identical in the secondary follicles and the small antral follicles.
The expression of MIS reduced as the antral cavity enlarged further. In large antral follicles, the expression of MIS mRNA dropped as compared with the small antral follicles; the numbers of cells expressing MIS were also reduced (Fig. 4D). MIS expression was high in some of the granulosa cells, but was conspicuously low or even absent in others. The mean staining intensity of MIS mRNA in large antral follicles was similar to the primordial follicles.

As seen in the human ovary, in the large antral follicles, although the MIS expression was low and heterogeneous, the cumulous cells stained more strongly for MIS mRNA as compared with the mural cells. Semiquantative analysis confirmed the observation where the expression of MIS in the cumulous cells was higher than that in the mural cells (not shown); however, the difference failed to reach statistical significance.

MIS expression was observed at very low levels or almost undetectable in the atetric follicles (Fig. 4E). Semiquantiatively, lowest expression of MIS was observed in these follicles.

No staining was visible in any of the sections hybridized with the sense probe demonstrating the specificity of staining (Fig. 4F). 

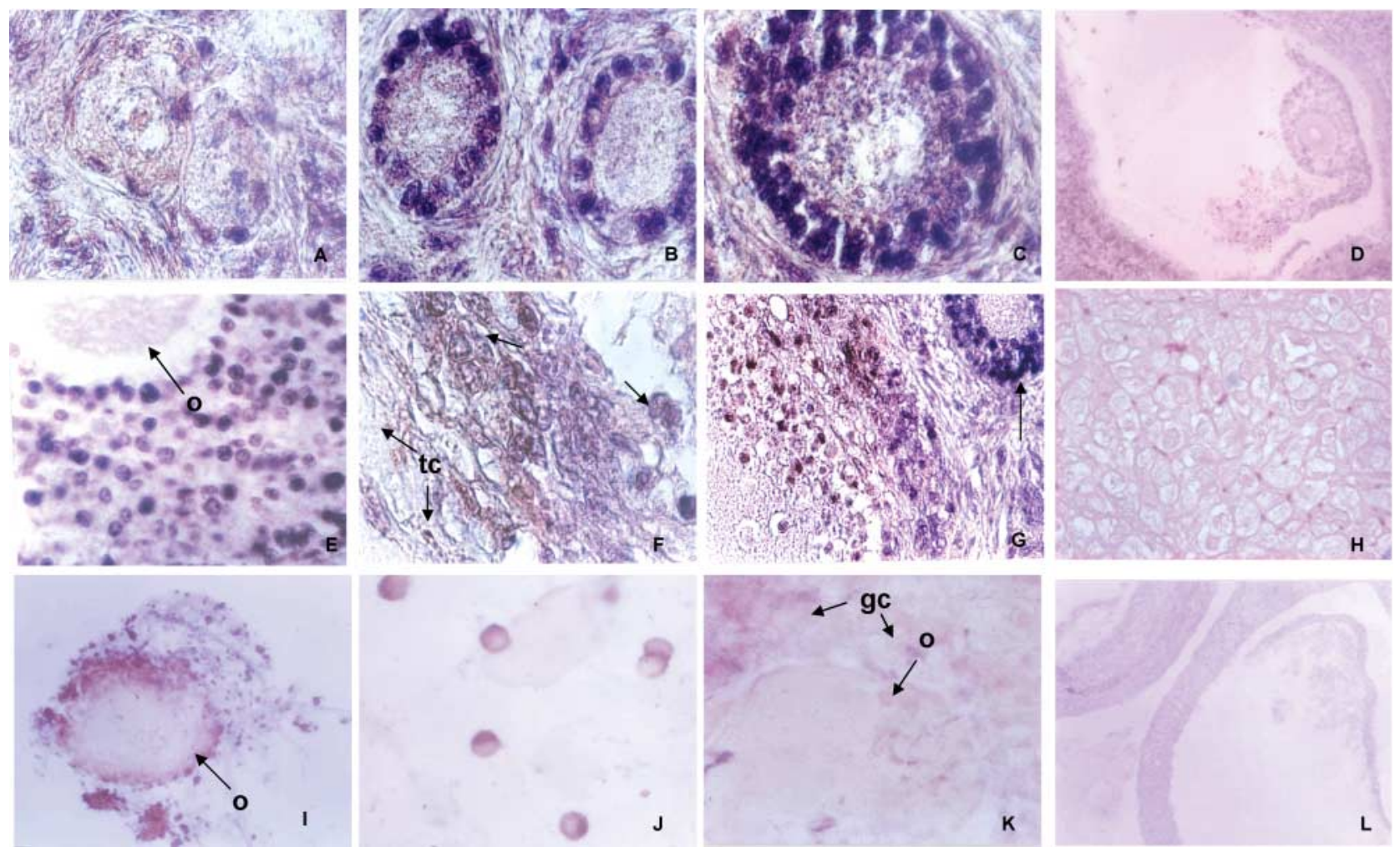

Figure 4 Cellular localization of Müllerian inhibiting substance (MIS) transcripts during folliculogenesis in the human ovary. (A) Primordial follicles. (B) Primary follicles. (C) Secondary follicle. (D) Large antral follicle. (E) Cumulous granulosa cells and the oocyte (o) of a large antral follicle. (F) The mural cells (arrow) and the theca (tc) layer of the same. Note the difference in the intensity of the staining of MIS mRNA in these cells. (G) An atetric follicle and a secondary follicle (arrow). (H) Corpus luteum that is negative for MIS mRNA. (I-K) Photographs of human cumulous oocyte complex (COC) stained for MIS mRNA: I is low magnification of the COC showing staining in the granulosa cells while the oocyte (o) has no staining; J is higher magnification of the granulosa cells; note the staining only in the periphery (cytoplasm) no nuclear signals are evident; $\mathrm{K}$ shows the granulosa cells (gc) closest to the oocyte (o) do not show MIS expression. Negative control is shown in L. Magnifications A, B, C, E, F, J and $\mathrm{K} 100 \times$ and digitized, D and L $20 \times, \mathrm{G}, \mathrm{H}$ and I $40 \times$.

\section{Discussion}

The results of the present study demonstrate that MIS mRNA is expressed in the granulosa cells of the fetal and the neonatal ovary, in adulthood, the pattern of expression varies according to the stages of folliculogenesis. During fetal life, MIS transcripts are expressed constitutively at low levels in the developing granulosa cells, a small number of oocytes express high levels of MIS transcripts. In the adult human and monkey ovaries, the granulosa cells express MIS mRNA at low levels in the primordial follicles, which increases in the primary and the preantral follicles, the expression drops with the formation of the antral cavity. MIS, at low levels, is persistently expressed in the granulosa cells of the large antral and the ovulatory follicles; the expression is further downregulated upon ovulation or at follicular atresia. The pattern of MIS expression during fetal life and in adulthood suggests its roles in follicular formation and the autocrine/paracrine regulation of adult folliculogenesis.

In the human fetal ovary, in situ hybridization revealed MIS expression in the granulosa cells at 13 weeks; the expression continues during follicular formation where MIS-positive granulosa cells envelope the oocytes to form the primordial follicles. To the best of our knowledge, this is the first report demonstrating the in situ localization of MIS in the fetal ovary and its association during folliculogenesis. Our findings corroborate the earlier results where MIS mRNA has been detected in the mid-trimester human fetal ovary; the levels have been estimated to be about $1.2 \%$ of the age-matched testicular tissue (Voutilainen \& Miller 1987). Contrasting these observations, immunoreactive MIS is not detectable in the human fetal ovaries in the second trimester, MISpositive granulosa cells are found in the primary follicles only after 30 weeks of development (Rajpert-De Meyets et al. 1999). It is possible that, during early gestation, the amount of MIS protein may be extremely low and/or may be rapidly utilized, the levels may rise during late gestation or as the follicle matures to the primary stage. Indeed, we observed an increase in MIS mRNA expression in the granulosa cells of the primary follicles as compared with the primordial follicles in the fetal and the neonatal ovary. 

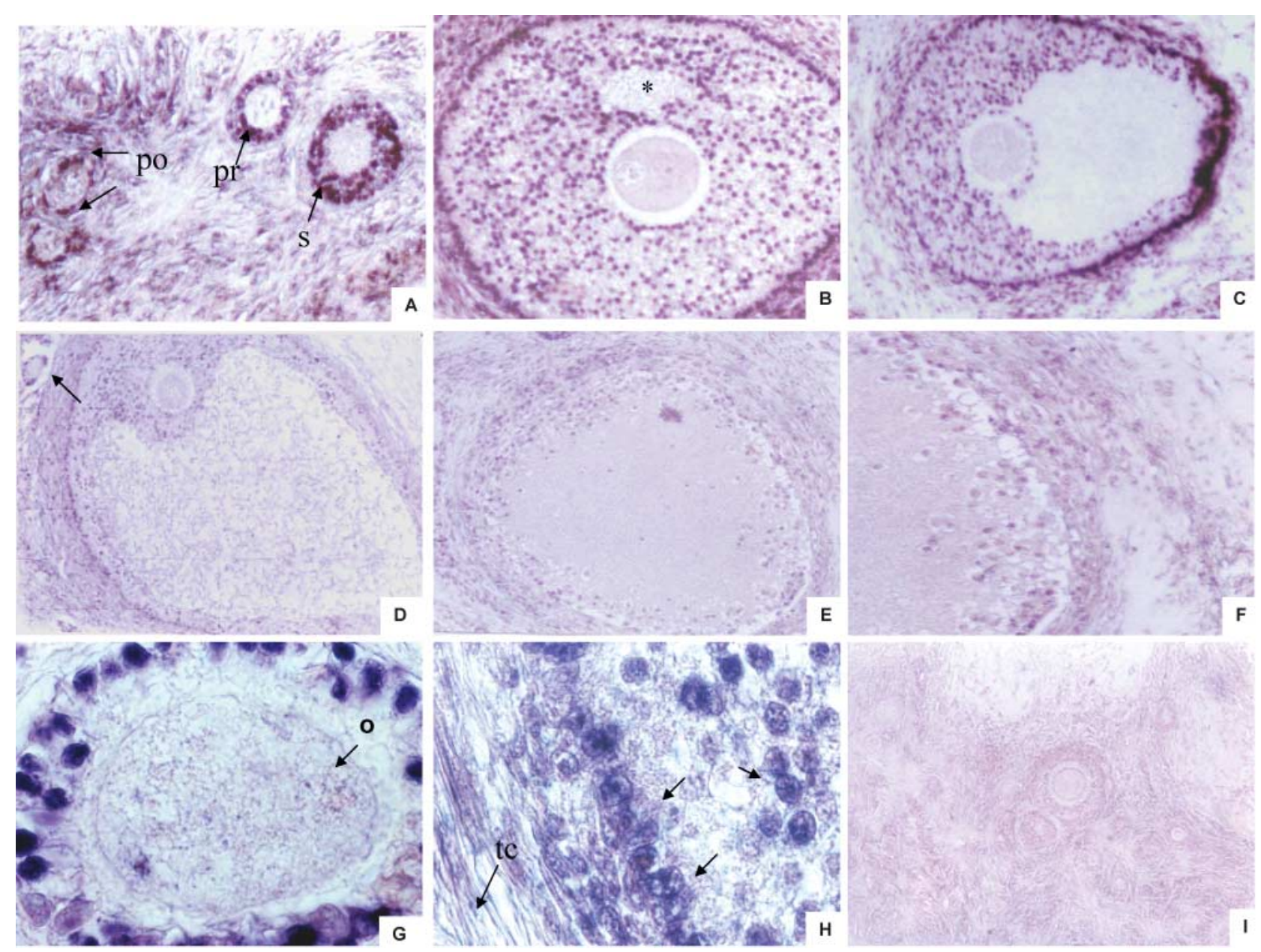

Figure 5 In situ hybridization for Müllerian inhibiting substance (MIS) transcripts in the bonnet monkey ovary. (A) Bonnet monkey ovary showing staining in the granulosa cells of primordial (po), primary (pr) and secondary (s) follicles. (B) Large preantral follicle (*antral cavity is just developed) showing strong MIS expression in the granulosa cells. (C) Antral follicle with reduced MIS expression. (D) Low levels of MIS mRNA in a large preovulatory follicle, compared with the primordial follicle (arrow). (E and F) Atetric follicle showing minimal MIS expression. (G) Cumulous granulosa cells and the oocyte (o) of a large antral follicle and $\mathrm{H}$ shows the mural cells (arrow) and the theca (tc) layer of the same. Note the difference in the intensity of the staining of MIS mRNA in granulosa cells between G and H. (I) Negative control.

At present, the precise functions of MIS in the fetal ovary are difficult to speculate in the absence of any supportive experimental data. MIS is absent in the fetal mouse ovary (Uneo et al. 1989b); the ovarian phenotype of the MIS knockout is essentially normal (Mishina et al. 1996). However, it is noteworthy that the fetal rodent ovary is responsive to MIS actions since female mice overexpressing MIS have streak ovaries owing to the loss of follicles during development (Behringer et al. 1990, Lyet et al. 1995, Josso et al. 1998).

A unique observation made in this study was the expression of MIS in a small subset of oocytes. MIS mRNA was detected at high levels in a small population of oocytes/oogonia of fetal human ovary; the frequency was estimated to be $\sim 1-3 \%$. These findings are novel and surprising since MIS has not been reported in the germ line of other mammalian species (Bezard et al. 1987, Uneo et al. 1989a, b, Hirobe et al. 1992, Durlinger et al. 2002a, Juengel et al. 2002). A striking feature of the MIS-positive oocytes was the absence of its association with the granulosa cells. Since oocytes not enveloped by the granulosa cells during development die by apoptosis (DePol et al. 1999, Modi et al. 2003), it is tempting to suggest that the MIS-expressing oocytes may be undergoing apoptosis. Indeed, in vitro incubation of fetal rat ovary with MIS leads to oocyte attrition (Vigier et al. 1987); gonadal dygenesis is observed in prenatal ovaries overexposed to MIS (Behringer et al. 1990, Lyet et al. 1995). Based on the observations made in this study and the experimental evidences, it appears that supraphysiological levels of MIS are detrimental to ovarian development; whether low levels of MIS are conducive to ovarian formation needs to be investigated.

The expression profiles of MIS in the adult human and monkey ovary are identical and comparable to that observed previously in other species (Bezard et al. 1987, 
Uneo et al. 1989a, Baarends et al. 1995, Juengel et al. 2002, Visser et al. 2006). Table 1 compares the results of different studies on cellular localization of MIS transcripts and protein in the adult cycling ovary. As evident, the results reported herein and those demonstrated by Stubbs et al. (2005) are comparable, the results differ with other studies. While in this study and that reported by Stubbs et al. (2005), MIS is detected in the granulosa cells of primordial follicles until ovulatory follicles; weak, but specific MIS transcripts and protein are detectable in the stromal cells. Contrasting these observations, previous studies have reported the absence of MIS in the primordial follicles and stroma (Bezard et al. 1987, Uneo et al. 1989a, Baarends et al. 1995, Juengel et al. 2002). To our knowledge, granulosa cells of the primordial follicles and the ovarian stroma as sites of MIS synthesis have not been reported earlier. There could be several explanations for such contrasting observations. Differences in sensitivity of the methods used, different fixatives, and staining protocols are some of the likely contributing factors. One striking observation reported by Stubbs et al. (2005) is the presence of MIS protein in the oocyte and theca cell cytoplasm, the mRNA for the same is not detectable in the same cell types (present study). The presence of MIS protein but not the mRNA in the theca cells may not be surprising, as the MIS receptor is expressed by these cells (Baarends et al. 1995); however, the expression of MIS in the oocyte is intriguing. MIS or its receptors have not been detected in the oocytes of the adult ovary of other species; additional studies are needed to define the origin and the role of MIS in the oocytes in the human ovary.

In the granulosa cells, MIS expression is dynamically regulated during folliculogenesis. Increase in MIS expression was evident in the primary and the secondary follicles as compared to the primordial follicles; little heterogeneity was evident in follicles within the same stage. MIS mRNA expression was downregulated as the antral cavity formed; this was more obvious in the granulosa cells of the large antral follicles. MIS transcripts were minimal in the atretic follicles. These results complement the recent observations where MIS protein has been immunolocalized predominantly in the granulosa cells of secondary and small antral follicles (Weenen et al. 2004, Stubbs et al. 2005) and indicate that the differential pattern of MIS protein expression during folliculogenesis is associated with the changes in MIS transcription and not due to differential utilization of the protein.

The changes in expression of MIS during various stages of early folliculogenesis may be an inherent property of the process and may not be under strict endocrine control. This is evident from the observation that MIS expression in the neonatal ovary, having spontaneously growing follicles until the early antral stage, was similar to that observed in adult human and monkey ovary. Interestingly, induction of MIS expression has also been observed in the primary follicles of day 4 neonatal mouse ovary (S Dharma, T Nandedkar, and D Modi, unpublished data). These observations tempt us to hypothesize that the dynamic regulation of MIS during early folliculogenesis may be under the control of some local autocrine and paracrine factors, the downregulation of MIS in the antral follicles may be in response to an endocrine signal.

The pattern of MIS transcription in the granulosa cells of human and monkey ovary reinforces the concept of stage-specific requirement of MIS in the process of follicular growth and maturation. In the primate ovary, increase in MIS transcription is observed in the granulosa cells of the growing (primary and secondary) follicles, the expression is downregulated upon their differentiation to antral follicles. This indicates that MIS may be required for follicle growth in the early phase; its downregulation is associated with differentiation. In support of these observations, it has been demonstrated that MIS is reported to promote the growth of human primordial follicles (Schmidt et al. 2005) and inhibition of differentiation of the preantral follicles (McGee et al. 2001) in vitro. In this context, it is also of interest to note that MIS transcription is extinguished in the granulosa cells only post-ovulation (as no nuclear MIS transcripts were evident in the granulosa cells obtained in the COC from an IVF cycle). Thus, it is likely that MIS may regulate granulosa cell proliferation while loss of MIS expression may lead to its differentiation. Thus, MIS may be

Table 1 Comparison of Müllerian inhibiting substance expression in the adult ovary.

\begin{tabular}{llllll}
\hline & $\begin{array}{l}\text { Uneo et al. (1989a), } \\
\text { Baarends et al. (1995) }\end{array}$ & $\begin{array}{l}\text { Durlinger et al. } \\
\mathbf{( 2 0 0 2 a , b )}\end{array}$ & Weenen et al. (2004) & Stubbs et al. (2005) & Present study \\
\hline Species & Murine & Mouse & Human & Human & Human \\
End product & mRNA & Protein & Protein & Protein & + \\
Primordial & - & - & - & + & ++ \\
Primary & $+/-$ & $+/-$ & + & ++ & + \\
Secondary/preantral & ++ & ++ & ++ & +++ & ++ \\
Small antral & +++ & +++ & +++ & + & + \\
Large antral/ovulatory & $+/-$ & + & - & + & + \\
Oocyte & - & - & - & + & + \\
Theca & - & - & - & + & + \\
Stroma & - & - & & & + \\
\hline
\end{tabular}


involved in the regulation of follicular growth and granulosa cell differentiation in the primate ovary.

An interesting observation made in this study is the difference in MIS expression in the cumulous versus mural granulosa cells of the large antral follicles. MIS transcripts were higher in the cumulus cells as compared to the mural ones. Although the differences were not statistically significant, the observations point towards disparate granulosa cell functions within the follicular compartment. Whether this is due to differential functional requirements of the cumulous and the mural cells of the preovulatory follicle, or of the differences in the responsiveness to an inhibitory signal, needs investigation.

In summary, the results of the present study in both human and monkeys strongly favor the regulatory roles of MIS in the folliculogenesis particularly in the process of follicular growth and differentiation. Based on the expression profiles and the results of some in vitro studies, it seems likely that the roles of MIS in ovarian functions in the rodents and primates may differ. MIS in the primate ovary may exert its effects in a larger temporal window initiating from the primordial follicle growth to terminal granulosa cell differentiation. The presence of MIS in the granulosa cells and a small subset of oocytes in the fetal ovary, point towards its additional role during fetal ovarian development that needs to be explored.

\section{Acknowledgements}

This work is a part of the PhD thesis work of D Modi. We express our gratitude to Dr S Gangal (ex Director, B J Wadia Hospital for Children) for kind guidance. We are extremely grateful to Dr L Vasudevan and Dr L Rajgopal (Anatomy Department, Seth GS Medical College) and Dr V Salvi (Obst and Gynec Department, Seth GS Medical College) for their help in collection of normal fetal gonads. We thank the staff of Histopathology department (Navrosjee Wadia Maternity Hospital) for the human ovarian tissue. Thanks to Dr A Malpani (Malpani Infertility Clinic, Mumbai) for the COC. The help extended by Dr S Sane and Dr S Sheikh during the study is gratefully acknowledged. The authors declare that there is no conflict of interest that would prejudice the impartiality of this scientific work.

\section{References}

Baarends WM, Uilenbroek JT, Kramer P, Hoogerbrugge JW, van Leeuwen EC, Themmen AP \& Grootegoede JA 1995 Anti-Müllerian hormone and anti-Müllerian hormone type II receptor messenger ribonucleic acid expression in rat ovaries during postnatal development, the estrous cycle and gonadotropin-induced follicle growth. Endocrinology 136 4951-4962.

Behringer RR, Cate RL, Froelick GJ, Palmiter RD \& Brinster RI 1990 Abnormal sexual development in transgenic mice chronically overexpressing Müllerian inhibiting substance. Nature 345 167-170.
Bezard J, Viger B, Tran D, Mauelon P \& Josso N 1987 Immunocytochemical study of anti-Müllerian hormone in sheep ovarian follicles during fetal and postnatal development. Journal of Reproduction and Fertility 80 509-561.

Cook CL, Siow Y, Brenner AG \& Fallat ME 2002 Relationship between serum Müllerian-inhibiting substance and other reproductive hormones in untreated women with polycystic ovary syndrome and normal women. Fertility and Sterility 77 141-146.

DePol A, Vacinia F, Forabosco A, Cavazzuti E \& Marzona L 1999 Apoptosis of germ cells during human prenatal oogenesis. Human Reproduction 112 2235-2241.

Durlinger Al, Kramer P, Karales B, de Jong FH, Uilenbroek JT, Grooegoed JA \& Themmen APN 1999 Control of primordial follicle recruitment by anti-Müllerian hormone in the mouse ovary. Endocrinology 142 5789-5796.

Durlinger AI, Gruijters MJ, Kramer P, Karels B, Kumar TR, Matzuk MM, Rose UM, de Jong FH, Uilenbroek JT, Grootegoed JA \& Themmen APN 2001 Anti-Müllerian hormone attenuates the effects of FSH on follicle development in the mouse ovary. Endocrinology $\mathbf{1 4 2}$ 4891-4899.

Durlinger AL, Visser JA \& Themmen AP 2002a Regulation of ovarian function: the role of anti-Müllerian hormone. Reproduction 124 601-609.

Durlinger Al, Gruijters MJ, Kramer P, Karels B, Ingraham HA, Nachtigal MW, Uilenbroek JT, Grootegoed JA \& Themmen APN $2002 b$ Anti-Müllerian hormone inhibits initiation of primordial follicle growth in the mouse ovary. Endocrinology 143 1076-1084.

Fallat ME, Cook C, Siow Y, Carrillo A \& Marra M 1997 Müllerian inhibiting substance in follicular fluid and serum: a comparison of patients with tubal factor infertility, polycystic ovarian syndrome and endometriosis. Fertility and Sterility 67 962-965.

Hirobe S, He WW, Lee MM \& Donahoe PK 1992 Müllerian inhibiting substance messenger ribonucleic acid expression in granulosa and Sertoli cells coincides with their mitotic activity. Endocrinology 131 854-862.

Josso N, Racine C, di Clemente N, Rey R \& Xavier F 1998 The role of anti-Müllerian hormone in gonadal development. Molecular and Cellular Endocrinology 145 3-7.

Juengel JL, Whale LJ, Wylde KA, Greenwood P, McNatty KP \& Eckery DC 2002 Expression of anti-Müllerian hormone in the brush tail possum (Trichosurus Vulpecula). Reproduction, Fertility, and Development 14 345-353.

Lee MM \& Donahoe PK 1993 Müllerian inhibiting substance: a gonadal hormone with multiple functions. Endocrine Reviews 14 152-164.

Lee MM, Gustafson ML, Ukiyama E, Donahoe PK, Maclaughlin W \& Keeping HS 1994 Developmental changes in Müllerian inhibiting substance in the Cynomolgus monkey, Maccaca fasicicularis. Journal of Clinical Endocrinology and Metabolism 78 615-621.

Lyet L, Louis F, Froest MG, Josso N, Behringer RR \& Viger B 1995 Ontogeny of reproductive abnormalities induced by deregulation of anti-Müllerian hormone expression in transgenic mice. Biology of Reproduction 52 444-454.

Malpani A, Malpani A \& Modi D 2002 The use of preimplantation genetic diagnosis in sex selection for family balancing in India. Reproductive Biomedicine Online 4 16-20.

McGee EA \& Hsueh AJ 2000 Initial and cyclic recruitment of ovarian follicles. Endocrine Reviews 21 200-214.

McGee E, Smith R, Spears N, Nachtigal MW, Inghram H \& Hsueh AJ 2001 Müllerian inhibiting substance induces growth of rat preantral follicles. Biology of Reproduction 64 293-298.

Mishina Y, Rey R, Finegold MJ, Matzuk MM, Cate RL \& Behringer RR 1996 Genetic analysis of the Müllerian inhibiting substance signal transduction pathway in mammalian sexual differentiation. Genes and Development 10 2577-2587.

Modi DM, Sane S \& Bhartiya D 2003 Accelerated germ cell apoptosis in sex chromosome aneuploid fetal human gonads. Molecular Human Reproduction 9 219-225. 
Modi D, Shah CA, Sachdeva G, Gadkar S, Bhartiya D \& Puri CP 2005 Ontogeny and cellular localization of SRY transcripts in the human testes and its detection in spermatozoa. Reproduction 130 603-613.

Patil V, Sachdeva G, Modi D, Katkam RR, Manjramkar DD, Hinduja I \& Puri CP 2005 Rab coupling protein (RCP): a novel target of progesterone action in primate endometrium. Journal of Molecular Endocrinology 35 357-372.

Penarrubia J, Fabregues F, Manau D, Creus M, Casals G, Casamitjana R, Carmona F, Vanrell JA \& Balasch J 2005 Basal and stimulation day 5 anti-Müllerian hormone serum concentrations as predictors of ovarian response and pregnancy in assisted reproductive technology cycles stimulated with gonadotropin-releasing hormone agonist gonadotropin treatment. Human Reproduction 20 915-922.

Piltonen T, Morin-Papunen L, Koivunen R, Perheentupa A, Ruokonen A \& Tapanainen JS 2005 Serum anti-Müllerian hormone levels remain high until late reproductive age and decrease during metformin therapy in women with polycystic ovary syndrome. Human Reproduction 20 1820-1826.

Rajpert-De Meyets E, Jorgsen N, Graem N, Muller J, Cate RL \& Skakkebaek NE 1999 Expression of anti-Müllerian hormone during normal and pathological gonadal development: association with differentiation of Sertoli and granulosa cells. Journal of Clinical Endocrinology and Metabolism 84 3834-3836.

Schmidt KL, Kryger-Baggesen N, Byskov AG \& Andersen CY 2005 AntiMüllerian hormone initiates growth of human primordial follicles in vitro. Molecular and Cellular Endocrinology 234 87-93.

Shah CA, Modi D, Sachdeva G, Gadkar S \& Puri CP 2005 Coexistence of intracellular and membrane-bound progesterone receptors in human testis. Journal of Clinical Endocrinology and Metabolism 90 474-483.

Stubbs SA, Hardy K, Da Silva-Buttkus P, Stark J, Webber LJ, Flanagan AM, Themmen APN, Visser JA, Groome NP \& Franks S 2005 Anti-Müllerian hormone protein expression is reduced during the initial stages of follicle development in human polycystic ovaries. Journal of Clinical Endocrinology and Metabolism 90 5536-5543.
Uneo S, Kuroda T, Maclaughlin DT, Ragin RC, Manganaro TF \& Donahoe PK 1989a Müllerian inhibiting substance in the adult rat ovary during various stages of the estrous cycle. Endocrinology 125 1060-1066.

Uneo S, Takashashi M, Manganaro TF, Ragin RC \& Donahoe PK 1989 b Cellular localization of Müllerian-inhibiting substance in the developing rat ovary. Endocrinology 124 1000-1006.

van Rooij IA, Broekmans FJ, Scheffer GJ, Looman CW, Habbema JD, de Jong FH, Themmen APN \& Te Velde ER 2005 Serum antiMüllerian hormone best reflect the reproductive decline with age in normal women with proven fertility: a longitudinal study. Fertility and Sterility 83 979-987.

Vigier B, Written F, Marge S, Tran D \& Josso N 1987 Purified bovine anti-Müllerian hormone induces a characteristic freemartin effect in fetal rat prospective ovaries exposed to it in vitro. Development 100 43-55.

Visser JA, de Jong FH, Laven JSE \& Themmen APN 2006 Anti-Müllerian hormone: a new marker for ovarian functions. Reproduction 131 1-9.

Voutilainen R \& Miller WL 1987 Human Müllerian inhibiting factor messenger ribonucleic acid is hormonally regulated in the fetal testis and adult granulosa cells. Molecular Endocrinology 1 604-608.

Weenen C, Laven JSE, von bergh ARM, Cranfield M, Groome NP, Visser JA, Kramer P, Fauser BC \& Themmen APN 2004 AntiMüllerian hormone expression pattern in the human ovary: potential implication for initial and cyclic follicle recruitment. Molecular Human Reproduction 10 77-83.

Received 9 March 2006

First decision 26 April 2006

Revised manuscript received 9 May 2006

Accepted 22 June 2006 